\title{
The fibonacci sequence and the jacobian matrix in food web models
}

\begin{abstract}
Impacts to a living community resulting from perturbation of a population variable can be predicted from the adjoint of the community (Jacobian) matrix, which is rendered in qualitative terms of complementary feedback cycles sequences which satisfy the Fibonacci sequence or its generalizations. This produces directed graphs in an absolute-feedback matrix that can clarify the sequence.
\end{abstract}

AMS classification numbers: 92D40, 11B39
Volume 3 Issue 2 - 2017

\author{
Anthony G Shannon \\ University of New South Wales, Australia
}

Correspondence: Anthony G Shannon, Warrane College, University of New South Wales, PO Box 123, Kensington NSW 1465, Australia,

Email t.shannon@warrane.unsw.edu.au, tshannon38@gmail.com

Received: September 08, 2017 | Published: October 04, 2017

\section{Introduction}

Biomathematics is at the heart of bioelectronics and the Fibonacci sequence goes back to the start of biomathematics. In the thirteenth century, Fibonacci (Leonardo Pisano) pondered the rate of reproduction in rabbits. Fibonacci's solution to the rate of reproduction in rabbits also gave birth, so to speak, to the discipline of population dynamics. Fibonacci's recurrence relation

$$
F_{t+2}=F_{t}+F_{t+1}
$$

Where $t$ is generation class, produces a number sequence for an exponentially expanding population-1, 1, 2, 3, 5, 8, 13, .., which projects, through time, mating pairs of rabbits and offspring over $t$ monthly generations. Over time, the ratio between successive generations of Fibonacci's rabbits $\left(n_{t} / n_{t-1}\right)$ converges to the golden ratio $\Phi(1.618 \ldots)$. The largest eigenvalue of this matrix equals $\Phi$ exactly, and is the exponentiated growth rate $(r)$ of the population, that is, $\lambda_{1}=e^{r}$. The next largest eigenvalue equals $\varphi(0.618 \ldots)$, where $\varphi=1 / \Phi$. We are concerned here with limits to growth for an ecological community that can be modelled electronically. The dynamics of $n$ interacting species can also be described by Lotka-Volterra equations ${ }^{1}$ which can be applied to biosensors, but we focus here on a qualitative analysis with a quantitative outline. The purpose of this paper is to use matrix and graph theory to highlight the presence of the Fibonacci sequence in the adjoint of Jacobian matrices which can arise in simple food web models. We then utilize an application to define terms and show the place of the Fibonacci sequence in the development of the main ideas and more complex models.

\section{Some matrix properties}

Fibonacci growth arises quite naturally in matrix representation. We can express the dynamics of Fibonacci growth in Leslie matrix form $^{2}$ and relate this to other known matrices and second order sequences.

$$
L_{n \times n}=\left[\begin{array}{cccccc}
0 & 1 & 1 & \ldots & 1 & 1 \\
1 & 0 & 0 & \ldots & 0 & 0 \\
0 & 1 & 0 & \ldots & 0 & 0 \\
& & & \ldots & & \\
0 & 0 & 0 & \ldots & 0 & 0 \\
0 & 0 & 0 & \ldots & 1 & 1
\end{array}\right]
$$

First row elements represent births of two offspring to each mating pair in generation $t$, and sub diagonal elements represent survival of each year class (here 100\%). The final diagonal element confers immortality to the population. When this element is zero, $L$ becomes the more familiar $Q$ matrix. $^{3}$ The Leslie matrix is also related to generalizations of the continued fraction algorithm. ${ }^{4}$ If we consider

$$
L_{n \times n}^{r}=\left[l_{i, j}^{(r)}\right] c
$$

Then it can be readily established that for $n>2$,

$$
\sum_{i=1}^{n} l_{i, j}^{(r)}= \begin{cases}F_{r+1} & j=1, \\ F_{r+2} & j>1,\end{cases}
$$

and

$$
\sum_{j=1}^{n} l_{i, j}^{(r)}=\left\{\begin{array}{cl}
U_{r+1, r+1}, & i=1, \\
U_{r+1, r}, & i=2,
\end{array}\right.
$$

In which $\left\{U_{r, m}\right\}$ is an integer sequence which satisfies the second order linear homogeneous recurrence relation (1.1) in the form

$$
U_{r . m}=U_{r, m-1}+U_{r, m-2}, m>2,
$$

With initial conditions $U_{r, 1}=1, U_{r, 2}=n-1$. When $n=2$, we get the Fibonacci sequence. ${ }^{5}$ The first few examples of this sequence are displayed in Table $1 .{ }^{10}$ As a further illustration, we can see that for $r=4$, and $n=5$,

$$
\left[\begin{array}{lllll}
0 & 1 & 1 & 1 & 0 \\
1 & 0 & 0 & 0 & 0 \\
0 & 1 & 0 & 0 & 0 \\
0 & 0 & 1 & 0 & 0 \\
0 & 0 & 0 & 1 & 1
\end{array}\right]^{4}=\left[\begin{array}{lllll}
2 & 3 & 3 & 3 & 3 \\
1 & 2 & 2 & 2 & 2 \\
1 & 1 & 1 & 1 & 1 \\
0 & 1 & 1 & 1 & 1 \\
1 & 1 & 1 & 1 & 1
\end{array}\right]
$$

So that, in turn,

$$
\begin{aligned}
& \sum_{i=1}^{5} l_{i, 1}^{(4)}=5=F_{5}, \\
& \sum_{i=1}^{5} l_{i, 2}^{(4)}=8=F_{6},
\end{aligned}
$$




$$
\begin{gathered}
\sum_{j=1}^{5} l_{1, j}^{(4)}=14=U_{5,5}, \\
\sum_{j=1}^{5} l_{2, j}^{(4)}=9=U_{5,4} .
\end{gathered}
$$

Note further, that if we treat (2.4) as a partial difference equation, then

$$
U_{r+1 . m}-U_{r, m-1}=F_{m-1}, m>2,
$$

and, furthermore,

$$
\begin{gathered}
\left\{U_{3 . m}\right\}=\left\{F_{m+1}\right\}, \\
\left\{U_{4 . m}\right\}=\left\{L_{m}\right\}, \\
\left\{U_{5 . m}\right\}=\left\{T_{m}\right\},
\end{gathered}
$$

$\left\{L_{m}\right\}$ is the well-known sequence of Lucas numbers, and $\left\{T_{m}\right\}$ is a sequence first investigated by Brother Alfred Brousseau. ${ }^{6}$

Table I $\left\{U_{\mathrm{r}, m}\right\}, r=3,4,5,6 ; m=1,2, \ldots, 7$

\begin{tabular}{llllllll}
\hline $\boldsymbol{m}$ & I & $\mathbf{2}$ & $\mathbf{3}$ & $\mathbf{4}$ & $\mathbf{5}$ & $\mathbf{6}$ & $\mathbf{7}$ \\
\hline$\left\{U_{3, m}\right\}$ & $\mathrm{I}$ & 2 & 3 & 5 & 8 & 13 & 21 \\
$\left\{U_{4, m}\right\}$ & $\mathrm{I}$ & 2 & 3 & 5 & 8 & 13 & 21 \\
$\left\{U_{5, m}\right\}$ & $\mathrm{I}$ & 4 & 5 & 9 & 14 & 23 & 37 \\
$\left\{U_{6, m}\right\}$ & $\mathrm{I}$ & 5 & 6 & 11 & 17 & 28 & 45
\end{tabular}

\section{Signed digraphs and qualitative analysis}

Signed digraphs portray relationships detailed in the community matrix by connecting system variables (or vertices) with links (or edges) ending in arrows $(\rightarrow)$, where there is a positive direct effect of one variable upon another, and filled circles ( $-\bullet)$, where the direct effect is negative. Thus, all possible (non neutral) pair-wise relationships can be described as predator prey or parasitism $(\bullet \rightarrow)$, mutualism $(\leftrightarrow)$, commensalism $(\rightarrow)$, interference competition $(\bullet \bullet \bullet)$, and amensalism $(-\bullet)$. Self-effects are shown by links originating and ending in the same variable and are typically negative $(\bullet \circlearrowright)$, as in self-regulated variables or those with density dependence, but can also be positive $(\circlearrowright)$ where variables are self-enhancing. Since it is difficult, or more often impossible, to actually measure all elements of the community matrix, a qualitative specification of a system's linkages may be the best that ecologists can do. Even so, simply knowing the signs of the interactions can provide important insights into the dynamics of complex systems. Counterintuitive behavior of a system often results from complex interactions, which can be revealed and understood through qualitative analyses ${ }^{7}$ For example, consider a model of the dynamics of snowshoe hare $(\mathrm{H})$ (boreal forest relative of Fibonacci's rabbits) interactions with vegetation (V), and a guild of predators $(\mathrm{P})$ including lynx and great horned owls, ${ }^{8}$ specified in both symbolic and qualitative form

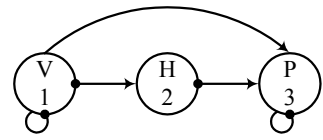

$$
A=\left[\begin{array}{ccc}
-a_{1,1} & -a_{1,2} & 0 \\
a_{2,1} & 0 & -a_{2,3} \\
a_{3,1} & a_{3,2} & -a_{3,3}
\end{array}\right] \quad{ }^{\circ} A=\left[\begin{array}{rrr}
-1 & -1 & 0 \\
1 & 0 & -1 \\
1 & 1 & -1
\end{array}\right]
$$

With associated adjoint matrices

$$
\begin{aligned}
\operatorname{adj}(-A) & =\left[\begin{array}{ccc}
a_{2,3} a_{3,2} & -a_{1,2} a_{3,3} & a_{1,2} a_{2,3} \\
a_{2,1} a_{3,3}-a_{2,3} a_{3,1} & a_{1,1} a_{3,3} & -a_{1,1} a_{2,3} \\
a_{2,1} a_{3,2} & a_{1,1} a_{3,2}-a_{1,2} a_{3,1} & a_{2,1} a_{1,2}
\end{array}\right] \\
\operatorname{adj}\left(-{ }^{\circ} A\right) & =\left[\begin{array}{rrr}
1 & -1 & 1 \\
0 & 1 & -1 \\
1 & 0 & 1
\end{array}\right]
\end{aligned}
$$

Where complementary feedback cycles in a response are opposite in sign, as in adj $\left(-A_{21}\right)$ in (3.2), then qualitative predictions are ambiguous and their sign determination depends upon knowledge of the relative strength of $a_{i j}$ interaction terms. With increasing system size and connectivity the number of complementary feedback cycles involved in response predictions grows not just exponentially, but factorially, and symbolic arguments can involve too many terms, perhaps hundreds, to interpret reasonably. We can account for the absolute number of terms in the adjoint matrix through use of the matrix permanent, denoted as ++ , in minors ( $\mathrm{min}$ ) of a community matrix specified only by the absolute value of its links; that is, by 1 's or 0 's only. This is an adjacency matrix that includes self-loops; denoted here as ${ }^{\circ}$ ), which, when transposed, gives the 'absolutefeedback matrix' $T$, where

$$
T_{i j}=+\min \cdot \mathrm{A}++^{\mathrm{T}}
$$

Completing the snowshoe-hare example, the absolute-feedback matrix then becomes

$$
T=\left[\begin{array}{lll}
1 & 1 & 1 \\
2 & 1 & 1 \\
1 & 2 & 1
\end{array}\right]
$$

Taking the ratio of each element of the adjoint to the absolutefeedback matrix, we obtain a 'weighted-predictions matrix' $W$ that scales the potential for sign determinacy in qualitative predictions of perturbation response. Adjoint matrix predictions with $W_{i j}$ values near one are highly reliable, while those near zero have a low potential for sign determinacy. The weighted predictions metric is essentially a signal-to-noise ratio with practical ecological applications; though not discussed further here, we refer the interested reader to. ${ }^{5}$

\section{The fibonacci sequence}

We can now see the special properties of the adjoint and absolutefeedback matrices and find complementary feedback cycles following the Fibonacci sequence. To clearly reveal its occurrence in matrix form, we consider a system much larger than the snowshoe-hare 
example. From a 10-variable straight-chain system, we can obtain the following adjoint matrix

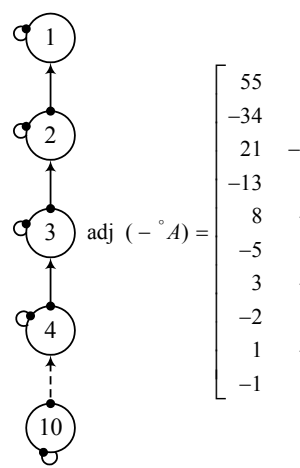

In this matrix, ignoring the signs, one observes the Fibonacci sequence along the first and last columns and rows. The left and right off-diagonal elements of the other columns are multiples of the first or last column, respectively, and the multipliers themselves are of the Fibonacci sequence. ${ }^{9}$ Considering the signs, where positive input propagates down the trophic chain (read down the columns), impacts alternate between positive and negative values, corresponding to a reversed Fibonacci sequence; that is, $n_{t-2}=n_{t}-n_{t-1}$, giving: ..., $34,-21,13,-8,5,-3,2,-1,1,0,1,1)$. One also observes negative starting values for the reversed sequence (i.e. ..., 55, $-34,21,-13$, $8,-5,3,-2,1,-1,0,-1,-1)$. As positive input propagates up the trophic chain, impacts are uniformly positive. The matrix is transdiagonally symmetrical. Since there is no countervailing feedback in this system, elements of the absolute-feedback matrix are equivalent to the absolute value of the adjoint matrix elements.

\section{Conclusion}

In biological terms, impacts from perturbations propagate through ecosystems via complementary feedback cycles that diminish in number away from the source of input according to the Fibonacci sequence. While Fibonacci's description of reproduction leads to a convergent value of $\Phi$ for a population's growth rate, so too does a convergent value of $\Phi$ (or $\varphi$ ) govern the proportion of complementary feedback cycles passed between adjacent members of an ecological community, and accordingly determines the reciprocal effect of neighbor upon neighbor. ${ }^{10}$ This can also be seen in simple models of the spread of infectious disease referred to earlier. We have outlined here the role of the Fibonacci sequence in the adjoint of community (Jacobian) matrices arising from simple food web models. Given that complementary feedback cycles can be positive or negative under different conditions and thereby cancel each other, we deduce the absolute-feedback matrix, the elements of which represent the absolute number of cycles in a response. This elucidation makes use of the permanent rather than the determinant of matrix minors. ${ }^{11}$ Both the determinant and permanent are recursively defined functions, and consequently give rise to the observed Fibonacci sequence even in qualitatively specified systems. These general observations can lead to more formal analysis by graph theorists. ${ }^{12}$

\section{Acknowledgements}

None.

\section{Conflict of interest}

The author declares no conflict of interest.

\section{References}

1. Dambacher JM, Rossignol PA. The golden rule of complementary feedback. ACM SIGSAM Bulletin. 2001;35(4):1-9.

2. Leslie PH. On the Use of Matrices in Certain Population Mathematics. Biometrika. 1945;33:183-212.

3. Shannon AG, Clarke JH, Hills LJ. Contingency relations for infectious diseases. In: M Witten editors. Mathematical Models in Medicine. 1988;2:823-833.

4. Shannon AG, Leon Bernstein. The Jacobi-Perron algorithm and the algebra of recursive sequences. Bulletin of the Australian Mathematical Society. 1973;8(2):261-277.

5. Atanassov Krassimir T, Anthony G Shannon. Combined 3-Fibonacci sequences from a new type. Notes on Number Theory and Discrete Mathematics. 2016;22(3):5-8.

6. Brousseau, Alfred Br. Exploring recurrent sequences. The Fibonacci Quarterly. 1963;1(2):81-83.

7. Dubeau François, Shannon AG. A Fibonacci model of infectious disease. The Fibonacci Quarterly. 1996;34(3):257-270.

8. Tyson Rebecca, Sheena Haines, Karen E Hodges. Modelling the Canada lynx and snowshoe hare population cycle: the role of specialist predators. Theoretical Ecology. 2010;3(2):97-111.

9. Dambacher JM, Li HW, Rossignol PA. Relevance of community structure in assessing indeterminacy of ecological predictions. Ecology. 2002;83:1372-1385.

10. Leyendekkers JV, Shannon AG. Some golden ratio generalized fibonacci and lucas sequences. Notes on Number Theory and Discrete Mathematics. 2016;22(1):33-41.

11. Miles EP. Generalized fibonacci numbers and associated matrices. American Mathematical Monthly. 1960;67(8):745-752.

12. Dambacher JM, Li HW, Wolff JO, et al. Parsimonious interpretation of the impact of vegetation, food, and predation on snowshoe hare. Oikos. 1999;84(3):530-532. 